\title{
EL PROFESOR Y EL ALUMNO. FIGURAS CLAVE EN EL PROCESO DE ENSEÑANZA-APRENDIZAJE DEL SISTEMA ACTUAL Y EN EL EEES ${ }^{1}$
}

\author{
María Jesús Sánchez y Alfredo Fernández-Sánchez, Universidad de Salamanca \\ Email:mjs@usal.es
}

\begin{abstract}
In this paper we examine the relationships established in the teaching and learning process. The main goals are to analyse the teacher's role and the requirements that he or she should meet (teacher training, methodological knowledge, etc.), and also to study the student's role and the problems related to this one caused by the current education system: overcrowding and absenteeism. These problems will disappear, in all likelihood, in the near future with the European guidelines on our education system. Keywords: teaching, learning, teacher, student, student's role, overcrowding, absenteeism.

Title in English: The Teacher and the Student. Key Figures in the Teaching-Learning Process in the Current System and in the EHEA

Resumen: En este trabajo se examinan las relaciones que se establecen en el proceso de enseñanza-aprendizaje. Los objetivos que se persiguen son analizar el papel que desempeña el profesor y los requisitos que debe cumplir (práctica docente, conocimiento metodológico, etc.). También el papel que desempeña el estudiante y los problemas que se relacionan con él causados por el sistema actual de educación: masificación y absentismo. Problemas que probablemente desaparecerán con la Convergencia Europea.

Palabras clave: enseñanza, aprendizaje, profesor, estudiante, papel del estudiante, masificación, absentismo.
\end{abstract}

\section{INTRODUCCIÓN}

Dentro del proceso de enseñanza-aprendizaje que se da en la actualidad, y que se dará en lo que se conoce como Espacio Europeo de Educación Superior (EEES), hay dos figuras de gran relevancia dentro de lo que se conoce como el "triángulo didáctico" (Bolívar, 2005; D’Amore y Fandiño Pinilla, 2002; Vera y Morales, 2005). Los tres vértices: saber, alumno y docente, interactúan en el seno de la estructura del sistema educativo y dan lugar a la intervención didáctica. En esta intervención destacan la figura del profesor y la del estudiante, las cuales se analizan detalladamente en este trabajo. Las dos son igualmente importantes y entre ellas se da una relación de mutua necesidad para que el proceso de enseñanza-aprendizaje tenga éxito. Estas dos figuras tienen un papel distinto

Date of reception: 31 May 2010

Date of acceptance: 8 November 2010

Odisea, ${ }^{\circ} 11$, ISSN 1578-3820, 2010, 259-268 
y complementario, y por supuesto diferentes objetivos. Así, el profesor, el cual utiliza sus competencias profesionales para facilitar el proceso de aprendizaje, tiene como objetivos, entre otros, fomentar la curiosidad en torno a la materia y a la cultura, estimular el interés y la curiosidad, crear actitudes críticas, diseñar tareas de estudio y aprendizaje autónomo coherentes con la evaluación (Morales Vallejo, 2005), etc. Es decir, preparar al alumno para aquello que la sociedad necesita y que le va exigir cuando acabe su formación, dotándole de un perfil profesional. Característica que representa una novedad con respecto al papel que se le venía asignando. El proceso de Convergencia con Europa conlleva cambios que afectan al sistema de enseñanza-aprendizaje y a las competencias del profesor universitario. Entre ellas se encuentran la enseñanza centrada en el alumno, quizá el enfoque que mejor responde a las directrices marcadas en Bolonia (Morales Vallejo, 2005), el manejo de las nuevas tecnologías, el diseño de la metodología de trabajo, la evaluación del proceso y del alumno, etc. (Fernández, 2005). Para que todo esto se lleve a cabo con éxito el profesor debe estar dispuesto a aceptar e interiorizar esta nueva situación. Como dice Argüelles Álvarez (2006) “al adoptar métodos pedagógicos más innovadores, el profesor debe estar preparado en lo que a la formación se refiere y dispuesto a afrontar nuevos retos, ..." (28). El estudiante, el cual construye su conocimiento mediante sus competencias cognitivas (http://orbita.starmedia.com/ constructivismo/constructivismo.htm), ha de tener como objetivos comprender, aprender y asimilar aquello que se trabaja dentro y fuera del aula en relación con la disciplina objeto de estudio, y desarrollarse paulatinamente hasta llegar a tener la máxima autonomía posible en el proceso de aprendizaje. Se convertirá, de este modo, en el centro del proceso construyendo el conocimiento, siendo crítico y actuando con responsabilidad en lo que respecta a su propio trabajo. En esto se diferencia necesariamente del papel que desempeña en la actualidad, en el que el profesor ocupa un puesto más prominente.

El profesor y el alumno pueden tener más o menos relevancia dependiendo del eje que se priorice dentro del triángulo didáctico: enseñar, aprender o formar (Vera y Morales, 2005). También puede variar dependiendo del tipo de actividad que se esté realizando en clase o del momento en que se halle el proceso educativo. Fundamentalmente, al principio, es el profesor el que carga con el peso de la clase hasta que el curso se pone en funcionamiento, se organizan las tareas y se conoce el método de trabajo. Posteriormente, la figura del estudiante se acrecienta si se siente involucrado en su propio proceso de aprendizaje, factor al que paulatinamente se le va concediendo una mayor importancia. Así, hasta que se magnifica, lo que suele coincidir con el final del cuatrimestre o con el término del año académico. Esto es fundamental en todas las disciplinas, pero quizá adquiera más importancia en el aprendizaje de una lengua extranjera en la que el estudiante llega a ser casi el protagonista.

Hay un buen número de investigaciones sobre la concepción del profesor universitario en la enseñanza y en el aprendizaje, lo que se relaciona con el modo de aproximación del estudiante a la materia (Anderson y Adams, 1992; McKeachie, 2002; Sherin 2002; Meléndez-Ferrer y Canquiz, 2003; Pérez Cañado, Casas Pedrosa, Ráez Padilla y Lancaster, 2007; Sánchez, 2005; Sanz Oro, 2005; Tigelaar, Dolmans, Wolfhagen y van der Vleuten, 2004; etc). Fruto de estas investigaciones es el trabajo de Boulton-Lewis, Smith, McCrindle, Burnett y Campbell (2001) de gran interés por la modernidad de ideas y por la 
extensa revisión que hacen estos autores de la concepción de los profesores frente al proceso educativo. Ésta se muestra en las diferentes investigaciones que analizan y de ellas se extraen las principales tendencias que se dan en la instrucción dependiendo de si se centra en el profesor y por lo tanto está orientada al contenido, de si se centra en el alumno y se orienta al aprendizaje, o de si es un continuo en el que se produce la integración con los estudiantes. Concluyen que la tendencia más adecuada es aquella que busca la interacción con el estudiante, la cual hace que éste desarrolle y alcance un mayor protagonismo. Como estos autores dicen "conceptions of teaching are best thought of in terms of a continuum of dominant conceptions ranging from teacher centred transmission of knowledge to a student centred focus involving transformation of the learners." (2001: 49). En este continuo la línea no es recta y unidireccional, ya que aunque ésta parta del profesor y se dirija al alumno también recorre el camino inverso dependiendo de las diversas actividades que se realizan en la clase y de la autonomía que va desarrollando el alumno. Esto es congruente con la idea ya expuesta sobre la importancia inicial del profesor, el cual progresivamente pasa a ocupar un puesto menos visible y central en el proceso de enseñanza-aprendizaje. Posición que es tomada poco a poco por el alumno. Se produce la integración y empatía con el alumnado que a veces recurre al profesor en busca de ayuda concediéndole, a pesar de estar éste eclipsado en beneficio de un mejor rendimiento del proceso de enseñanzaaprendizaje, el papel relevante que tiene.

Para que el proceso de aprendizaje se produzca como un continuo es absolutamente necesario una planificación exhaustiva de todas las asignaturas que componen el plan de estudios de la carrera estudiada y del papel que desempeña la asignatura que se imparte con respecto al resto de las asignaturas del curso (Argüelles Álvarez, 2006; Morales Vallejo, 2005). Es un proyecto que supone la colaboración y coordinación de todos los profesores y que requiere también la realización de una guía docente. En ella se deben recoger los objetivos, la metodología, las tareas a realizar, el tiempo que se va emplear en ellas y, por supuesto, tanto la evaluación de las actividades formativas que se llevan a cabo a diario como la final. Así mismo, se ha de meditar sobre la actitud de los estudiantes, su capacidad para comprender y elaborar, sus habilidades, las competencias que se deben dominar, etc. El conocimiento de los principios psicopedagógicos y el contar con una formación continua ayuda en esta tarea de planificación a la vez que contribuye a perfilar el papel que debe desempeñar el profesor y el alumno, interés primordial de este trabajo.

\section{MODELO DE PROFESOR UNIVERSITARIO Y SUS TAREAS}

El modelo de profesor universitario que demanda la universidad, de acuerdo con las funciones que se asignan a esta institución y cuyo correcto ejercicio requiere la creación de conocimiento mediante la investigación, la constante actualización del mismo a través del estudio y su aplicación a una enseñanza de calidad, es la de un profesional. Este concepto se puede entender con la definición de Cruz Tomé (1996: 1): "Ser profesional supone poseer un conjunto de conocimientos, destrezas y actitudes que le hacen eficiente en el desempeño de su tarea." Parte de esta preparación, es decir, las destrezas y actitudes, se consiguen principalmente cuando al terminar la carrera uno se matricula en el Curso de Aptitud Pedagógica. Este curso, o aquéllos que ya se están adoptando (Máster en Profesor 
de Educación Secundaria Obligatoria y Bachillerato, Formación Profesional y Enseñanza de Idiomas), suponen una buena ayuda ya que cuando uno se gradúa y comienza a impartir docencia normalmente tiene mucho conocimiento que comunicar, pero le faltan recursos sobre el modo adecuado de hacerlo. En la mayor parte de los casos, debido a como se ha recibido la propia educación, en íntima relación con el estilo de la universidad en la que se han cursado los estudios, apenas se sabe cómo llevar a cabo el proceso de enseñanza. Esta ayuda inicial y las actitudes que se desarrollan con la práctica colaboran en gran medida al modo adecuado de impartir nuestras clases pero son insuficientes para seguir sin más apoyo nuestra vida docente.

Es necesario, además de útil, una formación continua que permita al profesor estar al día de las experiencias e innovaciones de otros profesionales y que le proporcione el marco adecuado para la reflexión e interiorización de las nuevas exigencias. En este sentido hay que recordar el esfuerzo que hoy en día realizan un gran número de universidades para proporcionar a su profesorado cursos para la actualización metodológica y también sobre las nuevas tecnologías. Con esta ayuda el profesor aprende a manejar diferentes recursos que puede emplear de cara a la docencia (ej. aplicaciones informáticas: Power Point, pizarras digitales, tecnología de video, etc.) Pero sobre todo aprende o recuerda ciertas estrategias que pueden contribuir a potenciar la creatividad, a dar un aire más interesante al material y a sus propias explicaciones, etc. y dedica un espacio de tiempo a comunicar sus logros o preocupaciones docentes con aquéllos que están involucrados en el mismo proceso. Esta comprensión mutua le hace más receptivo, lo que le permite una mayor asimilación de los modos más eficaces de actuar en el aula. La puesta en práctica de nuevas técnicas y de ideas metodológicas sobre motivación, organización, evaluación, etc. repercute en una mejor captación del contenido por parte del estudiante, al mismo tiempo que da lugar a un nuevo rol en éste. De ahora en adelante el discente, de acuerdo con los enfoques de enseñanza más innovadores, se verá involucrado en el proceso de aprendizaje lo que con toda probabilidad hará que participe voluntaria y activamente.

Así, a través de los distintos curso de contenido docente y metodológico, junto a los que versan más con el contenido y su investigación (ej.: seminarios, congresos, etc.) el profesor obtiene una formación continua que le convierte progresivamente en un profesional de la enseñanza. Es un proceso lento que requiere práctica y un buen apoyo institucional, pero es muy gratificante poder llegar a contar con las características que poseen los buenos profesores y que señala Cruz Tomé en su investigación (1996: 6). Aunque no están pensadas para el EEES serán igualmente de gran utilidad. Son las siguientes:

1. El profesor universitario debe ser un especialista, al más alto nivel, de su área de conocimiento. Esta especialización tiene que estar vinculada a la investigación sobre el contenido de su materia y la docencia de la misma.

2. Formado profesionalmente en cada una de las tareas que tiene que desempeñar, principalmente: investigación, docencia y gestión.

3. Motivado por la investigación y docencia de su asignatura. Con entusiasmo, interés y vocación por ella.

4. Poseer ciertos rasgos de personalidad que la tarea docente e investigadora exigen. Entre otros: paciencia, tolerancia, empatía, justicia, apertura, disponibilidad, interés 
por los otros, adaptación, creatividad, curiosidad intelectual, flexibilidad y sentido del humor.

5. Poseer ciertas habilidades personales básicas. Entre éstas: facilidad para las relaciones interpersonales, habilidades para la comunicación, control del estrés, tolerancia a la frustración, asertividad, etc.

6. Poseer habilidades docentes específicas. Tales como: organización y estructuración de los conocimientos a impartir y de las condiciones de su aprendizaje, planificación a largo y a corto plazo de las actividades docentes y discentes, claridad expositiva, presentación de los contenidos de manera que despierte el interés de los estudiantes y promueva el deseo de seguir aprendiendo, fomento del pensamiento independiente y crítico, organizador y gestor de situaciones y recursos de aprendizaje, conocimiento de alternativas metodológicas de enseñanza, justicia en las evaluaciones, manejo eficaz del tiempo, expresividad ...

7. Poseer una actitud crítica y reflexiva sobre su propia actuación como profesor.

La posesión de todas estas características le convierten a uno en el profesor especializado ideal. Es decir, aquél que se tiene en meta, que se desea llegar a ser y que requieren los tiempos actuales en detrimento de la formación integral que se solicitaba antiguamente.

A veces no es posible conseguir ser un profesor especializado debido a factores de diversa naturaleza y que son ajenos al docente. Esta primera característica, necesaria para el buen quehacer docente y por ello de gran coherencia el que se pida, en algunos casos no se da. Esto es debido a que el profesor muchas veces no tiene la opción de elegir su docencia, lo que origina, si ésta no coincide con su investigación, que el docente se vea en la difícil situación de estar atrapado entre dos mundos. Además, el hecho de que la docencia que se imparte y de que incluso los contenidos docentes puedan variar con cada curso académico no fomenta el que se hagan cambios en pro de una unificación entre lo que se investiga y lo que se enseña. La coincidencia en este primer apartado entre docencia e investigación debería fomentarse, en la medida de lo posible, para que ambas actividades caminen en la misma dirección. Éste es un hecho decisivo en el éxito de la práctica docente.

En cuanto a la segunda, tercera y séptima características éstas pueden verse favorecidas, en parte, por el apoyo institucional. Éste se ve plasmado en cursos didácticos, en el apoyo económico que posibilita el intercambio con otros colegas (ej. ayudas de asistencia a congresos y seminarios, ayudas para estancias en otras universidades, etc.) y en contar con la infraestructura adecuada. Estas características, las cuales se consideran imprescindibles en la vida de un profesional, por lo general, se dan en mayor o menor medida dependiendo de la importancia que la propia universidad les conceda y de si se da un reconocimiento oficial, por su parte, de la calidad docente e investigadora que se lleva a cabo en su seno. Del mismo modo, las habilidades que se mencionan en el sexto apartado son las que se intentan fomentar más directamente en los cursos de metodología. Se pueden poner en práctica fácilmente, con ciertos conocimientos y reflexión sobre el proceso de enseñanza, y desde luego son imprescindibles para el buen funcionamiento de la clase. Desarrollar estas habilidades no supone un coste elevado para el profesor ya que coinciden en gran parte con las tareas básicas de planificación que se desempeñan en el proceso de enseñanza-aprendizaje. 
El cuarto y quinto apartado aluden, más bien, a características personales. Éstas, al menos las del cuarto punto, se pueden desarrollar con constancia, voluntad, interés y siempre que haya una atmósfera que demande este tipo de comportamiento que sea sensible a los rasgos aquí expuestos. En lo que respecta al quinto apartado hay que decir que también es posible mejorar con la reflexión, comunicación, etc., pero a mi modo de ver es más difícil. Se puede dar un cambio en nuestra personalidad gracias a la educación, pero hay que reconocer que las características personales al ser rasgos consolidados son más reacios al cambio.

Se concluye, que aunque no siempre se den las mejores condiciones para promover y llegar a ser el profesor ideal, debido: a) en parte al problema que se ha planteado en el que la docencia e investigación no siempre van por el mismo derrotero, b) a ciertos rasgos de personalidad difícilmente cambiables c) y a otros problemas que no parten del profesor y que tienen que ver con la noosfera (D’Amore y Fandiño Pinilla, 2002) como pueden ser: la masificación del alumnado, la disposición de las aulas, los padres, directivas ministeriales, etc., cierta ayuda institucional y permanente junto con la predisposición favorable para el buen ejercicio de esta profesión pueden tener como resultado un profesor coherente, reflexivo e interesado en una formación continua. De ser así, se auguran buenos resultados tanto para la enseñanza como para el aprendizaje, superando así las innumerables trabas que uno se encuentra día a día en el ejercicio de esta profesión entre las que se pueden contar programas demasiado amplios, espacios inadecuados, descompensación en el reconocimiento de méritos docentes e investigadores, etc.

\section{TIPO DE ALUMNOS Y APRENDIZAJE DE LA MATERIA}

En la labor de meditación sobre las características de los alumnos y sobre aspectos que inciden en el aprendizaje de la materia hay que constatar el problema con el que nos encontramos hoy en día en algunas disciplinas y que probablemente desaparecerán con la adaptación al EEES: aulas masificadas. Éste es un condicionante negativo en cualquier disciplina que se imparta y mucho más grave cuando se trata de un idioma extranjero. Para luchar contra él es necesario emplear los mejores recursos con los que se cuente. Exista o no este problema lo que aquí se propone porque se sabe que funciona, gracias a la experiencia docente, es favorecer siempre que la materia sea adecuada el trabajo en grupos pequeños. Se aconseja, si se trata de un idioma extranjero, un método esencialmente comunicativo porque se centra en el estudiante y le involucra en el proceso de enseñanza (Pica, 2000).

El trabajo con grupos pequeños se ha de planificar muy bien con unas directivas muy claras sobre su realización. Si no se hace así se corre el riesgo de que en cada grupo trabaje un solo estudiante y los demás se amparen en su trabajo, pasándole a veces desapercibida al profesor la aportación individual de cada uno al grupo. La importancia de la correcta realización del mismo se debe a que lo que importa en muchos casos, especialmente cuando se aprende una lengua extranjera, no es lo que se entrega como resultado sino el proceso seguido y el intercambio lingüístico llevado a cabo. Para que todos los estudiantes cooperen se necesita que el profesor realice un trabajo previo en el que se especifiquen claramente las características requeridas. Entre éstas se encuentran las siguientes:

1. Que cuenten con el material adecuado. 
2. Que conozcan muy bien el proceso que han de seguir.

3. Que sepan que van a conseguir con ese trabajo.

4. Que conozcan las expectativas del profesor frente a ese trabajo.

5. Que los grupos guarden un equilibrio.

6. Que el profesor al moverse entre los estudiantes resulte una ayuda y no una amenaza.

7. Que el tiempo que se va a dedicar a esa actividad esté bien controlado para no fomentar, así, la frustración si se peca por defecto o el aburrimiento si se peca por exceso.

8. Que tengan claro como se les va a evaluar.

Otro de los problemas serios que nos encontramos hoy en la universidad y que se ha de tener en mente para poderlo combatir es el absentismo. Es posible que con la Convergencia Europea este problema desaparezca si se da la esperada reducción de estudiantes por grupo y si la asistencia pasa a ser obligatoria (cosa que de momento no es más que un deseo y una especulación), pero que hoy hay que contar con él. Como comenta Hernández Hernández (1989: 306) "Para todos los efectos el alumno 'oficial' es un alumno 'libre' “. El problema radica en que la universidad española concede total libertad al alumno para asistir o no a clase, a diferencia de otros países en que la asistencia es obligatoria (ej. Inglaterra, EE.UU., etc.). Esto tiene como consecuencia faltas esporádicas a clase por parte de distintos alumnos. El resultado, se les preste atención o no a éstos, es un entorpecimiento en el progreso de la clase; especialmente en clases de naturaleza práctica como las de idiomas extranjeros. Se insta a recurrir al uso de los aspectos psicopedagógicos porque a través de éstos se crea el interés y la motivación que el alumno necesita para considerar necesaria la asistencia a clase, lográndose así el deseo de acudir a ella y, probablemente, una sensación de bienestar en el aula. Con la Convergencia Europea la asistencia a clase no será algo deseable, se convertirá en una práctica impulsada si no por la obligación sí por la reducción del número de alumnos por grupo.

Una forma de combatir el absentismo que se da en la actualidad y de favorecer el deseo de asistir a clase puede ser a través de la asignación de uno o más trabajos, dependiendo de la duración de la asignatura (cuatrimestral o anual), que sea o sean cada vez más complejos. Esto hará sentir al alumno que para su cumplimiento son fundamentales las explicaciones, los ejercicios e intercambios que se llevan a cabo en el aula. Designar un porcentaje de la nota a diversas actividades realizadas en clase hace que el alumno se involucre, se esfuerce por ir ganando esos puntos y asista con regularidad. El procedimiento que se puede seguir en casi todas las ocasiones es el siguiente:

- Establecimiento de grupos pequeños formados normalmente al azar (ej. asignar un número a cada estudiante y formar un grupo con los múltiplos de un determinado número o con los primeros números pares, etc.) En otras ocasiones, dependiendo de la dificultad del trabajo, cohesión del grupo, etc. se puede dar la opción de trabajar con el compañero o compañeros que se desee, señalándose el límite máximo de tres 
o cuatro estudiantes para posibilitar una mayor discusión e intervención de cada miembro en el grupo.

- Asignación de días y horas para la realización de un proyecto con el tema elegido por los discentes, entre varias opciones, o buscado de acuerdo con ciertos criterios previamente establecidos: tipo de texto, estructura, longitud, etc.

Al final del cuatrimestre o de un cierto periodo fijado con anterioridad tienen como requisito la entrega de un trabajo o trabajos donde se muestra, se explica y se analiza la labor llevada a cabo.

Esta actividad resulta muy satisfactoria ya que los alumnos cuentan espontáneamente con el profesor si le necesitan. De lo contrario, se interrogan unos a otros, buscan páginas web y otra información fácil de encontrar, etc. Se percibe como un tiempo dedicado al trabajo personal con el fin de aprender y en el que se les nota a gusto avanzando en el trabajo encomendado. Hay que decir que, por lo general, cuando se trabaja de este modo los alumnos cumplen perfectamente con las dos cosas que se les pide, las cuales son la asistencia regular a clase y la entrega del trabajo en su debido momento. El trasfondo de este tipo de actividad es aplicable al resto del trabajo que se hace en clase. Para ello, sólo hay que esforzarse en una planificación coherente en la que se involucre al alumno en su propio aprendizaje y donde el interés haga que éste tenga un papel activo.

\section{CONCLUSIONES}

En consonancia con las funciones que se asignan a la Universidad surge el modelo ideal de profesor que ésta demanda y que se conforma a base de las características ya mencionadas (Cruz Tomé, 1996: 6), las cuales son válidas para el sistema de enseñanza-aprendizaje actual y el venidero. La clave para salir con éxito está en la administración correcta de los aspectos psicopedagógicos, a través de los cuales se involucra y se crea la motivación que el alumno necesita para considerar necesaria la asistencia a clase. El profesor experimentado, conocedor de los diferentes recursos que tiene a mano, subsana las dificultades utilizando trabajo en grupos pequeños, independiente, etc., lo que hace que se alcance el nivel adecuado en cada curso. El trabajo en grupos pequeños, que motiva e involucra al estudiante, se considera fundamental aún en ausencia de los condicionamientos negativos comentados que se dan en la actualidad porque promueve una mayor participación en el proceso de aprendizaje. Las nuevas metodologías han dado un vuelco a la manera de conceptualizar al docente, a éste se le considera ahora como un mediador y facilitador del aprendizaje. Idea que necesariamente repercute en la manera de aprender, proceso que en este nuevo paradigma se centra en el estudiante.

\section{REFERENCIAS BIBLIOGRÁFICAS}

Anderson, J. A. y M. AdAms. 1992. "Acknowledging the learning styles of diverse populations: Implications for instructional design." New Directions for Teaching and Learning 49: 19-33. 
Argüelles Álvarez, I. 2006. “El Espacio Europeo de Educación Superior y la adaptación de las asignaturas a los nuevos enfoques pedagógicos: inglés técnico en la EUIT de Telecomunicación.” Greta 14(1-2): 27-35.

Bolívar, A. 2005. "Conocimiento didáctico del contenido y didácticas específicas." Profesorado. Revista de Currículum y Formación del Profesorado 9(2). http://www. ugr.es/local/recfpro/Rev92ART6.pdf

Boulton-Lewis, G. M., D. J. H. Smith, A. R. McCrindle, P. C. Burnett y K. J. CAmPBELL. 2001. "Secondary teacher's conceptions of teaching and learning." Learning and Instruction 11: 35-51.

Cruz Tomé, Ma A. DE LA. 1996. "La profesión de Profesor Universitario.” Manuscrito sin publicar presentado en el curso El Diseño de Proyectos de Innovación Docente en la Universidad de Salamanca. 4-5 de Julio, 2000.

D’Amore, B. y M. I. Fandiño Pinilla. 2002. “Un acercamiento analítico al 'triángulo de la didáctica' ". Educación Matemática 14(1): 48-61.

FERnÁndez, A. 2005. Nuevas metodologías docentes. Talleres de formación del profesorado de la UPM para la convergencia europea.

Hernández Hernández, P. 1989. "El Proyecto docente del profesor universitario. Diseñar y enseñar." Teorías y técnicas de la programación y del proyecto docente. Universidad de La Laguna: ICE. 301-326.

McKeachie, W. J. 2002. Teaching Tips. Boston: University of Michigan.

Melendez-Ferrer, L. y L. Canquiz. 2003. "La actitud del profesor universitario desde una perspectiva pedagógica, en el marco de la relación universidad-empresa." Revista de Pedagogía 24(71): 417-445.

Morales VAllejo, P. 2005. "Implicaciones para el professor de una enseñanza centrada en el alumno." Universidad Pontificia de Comillas, 15 de marzo.

Pérez Cañado, A., V. Casas Pedrosa, J. Ráez Padilla y N. K. Lancaster. 2007. “El aprendizaje cooperative en el sistema ECTS." II Jornadas de Trabajo sobre Experiencias Piloto EEES en la Universidades Andaluzas, Granada 30-31 de octubre.

PICA, T. 2000. "Tradition and transition in English language teaching and methodology." System 28: 1-18.

“Qué es el constructivismo?” http://orbita.starmedia.com/ constructivismo/constructivismo.htm

SÁnchez, L. 2005. "Concepciones de aprendizaje de profesores universitarios y profesionales no docentes: Un estudio comparativo." Anales de Psicología 21(2): 231-243.

SAnz ORO, R. 2005. "Integración del estudiante en el sistema universitario. La tutoría." Cuadernos de Integración Europea 2: 69-95. 
Sherin, M. G. 2002. "When teaching becomes learning." Cognition and Instruction 20(2): 119-150.

Tigelaar, D. E. H., D. H. J. M. Dolmans, I. H. A. P. Wolfhagen y C. P. M. van der VLEUtEN. 2004. "The development and validation of a framework for teaching competencias in higher education." Higher Education 48(2): 253-268.

Vera, M. y F. Morales 2005. "Propuesta de un modelo didáctico para la elaboración de un software educativo para la enseñanza del cálculo integral." Acción Pedagógica 14: 50-57. 